Highly Energetic Physical Processes and

Mechanisms for Emission from Astrophysical Plasmas

IAU Symposium, Vol. 195, 2000

P. C. H. Martens, S. Tsuruta, and M. A. Weber, eds.

\title{
Hypernovae: SNe 1997ef, 1998bw, and 1997cy
}

T. Nakamura and K. Maeda

Department of Astronomy and Research Center for the Early Universe, University of Tokyo, Japan

K. Iwamoto, T. Suzuki, K. Nomoto, and P. A. Mazzali ${ }^{1}$

Department of Physics, College of Science and Technology, Nihon University, Japan

M. Turatto

Osservatorio Astronomico di Padova, vicolo dell'Osservatorio, Padova, Italy

I. J. Danziger

Osservatorio Astronomico di Trieste, via G. B. Tiepolo, Trieste, Italy

F. Patat

European Southern Observatory, Garching, Germany

\begin{abstract}
.
We discuss the properties of the very energetic Type Ic supernovae (SNe Ic) 1998bw and 1997ef, and of Type IIn supernova (SN IIn) 1997cy. SNe Ic 1998bw and 1997ef are characterized by their large luminosity and very broad spectral features. Their observed properties can be explained if they are very energetic SN explosions $\left(E_{\mathrm{K}} \gtrsim 1 \times 10^{52} \mathrm{erg}\right)$, originating probably from the core collapse of the bare $\mathrm{C}+\mathrm{O}$ cores of massive stars $\left(\sim 30-40 M_{\odot}\right)$. At late times, both the light curve and the spectra suggest that the explosion may have been asymmetric; this may help us understand the claimed connection with GRBs. Type IIn SN 1997cy is even more luminous than SN 998bw, and the light curve declines more slowly than the ${ }^{56}$ Co decay. We model such a light curve with circumstellar interaction, which requires the explosion energy of $\sim 5 \times 10^{52} \mathrm{erg}$. Because these kinetic energies of explosion are much larger than in normal core-collapse SNe, we call objects like these SNe "hypernovae".
\end{abstract}

\footnotetext{
${ }^{1}$ Also at: Osservatorio Astronomico di Trieste, via G. B. Tiepolo, Trieste, Italy
} 


\section{Introduction}

The kinetic energies of core-collapse supernovae, i.e., Type II and Ib/c supernovae (SNe II, Ib/c), are fundamentally important quantities in relation to the explosion mechanism, impact on interstellar medium, and nucleosynthesis. In this paper, we use $E_{\mathrm{K}}$ as the terminal kinetic energy of the ejecta. Typically, $E_{\mathrm{K}} \sim 1 \times 10^{51} \mathrm{erg}$, and a good example is SN 1987A in the Large Magellanic Cloud, whose energy is estimated to be $E_{\mathrm{K}}=(1.0-1.5) \times 10^{51} \mathrm{erg}$ from its early light curve and spectra (e.g., see Shigeyama \& Nomoto 1990).

However, SN Ic 1998bw clearly demonstrates that this is not always the case. This supernova is almost 10 times brighter than the typical SN Ic 1994I, and its spectra show an unusually broad feature, which suggests a large explosion energy. More interestingly, SN 1998bw was discovered in the error box of the gamma-ray burst GRB 980425 one day after this GRB, which suggests a connection between these two events.

Other candidates for energetic supernovae, which we call "hypernovae" if $E_{\mathrm{K}} \gtrsim 10^{52} \mathrm{erg}$ (to distinguish from canonical supernovae), have been discovered recently. SN Ic 1997ef shows very broad spectral features similar to SN $1998 \mathrm{bw}$. The Type IIn supernova (SN IIn) 1997cy, which might have been associated with GRB 970514, is unusually bright (Germany et al. 2000; Turatto et al. 2000). In this paper, we study these three objects, SNeIc 1997ef, 1998bw, and SNIIn $1997 \mathrm{cy}$, as hypernova candidates.

\section{SN 1997ef}

SN 1997ef has been noticed for its unique light curve and spectra. At early times, the spectra were dominated by broad oxygen and iron absorption lines but did not show any clear feature of hydrogen or helium (Garnavich et al. 1997; Filippenko et al. 1997), which led us to classify SN 1997ef as an SN Ic. The most striking and peculiar feature of the spectra of SN 1997ef is the broadness of the line features, which suggests that SN 1997ef may have a large explosion energy. A possible connection with a GRB was also suggested for SN 1997ef: GRB 971115 appears, in fact, to be compatible with SN 1997ef in the position and time occurrence of the SN (Wang \& Wheeler 1998).

In order to clarify whether SN 1997ef is indeed a hypernova, we constructed hydrodynamical models for an ordinary SN Ic and for a hypernova (Iwamoto et al. 2000). For the ordinary SNIc (model CO60), a C+O star with a mass $M_{\mathrm{CO}}=6.0 M_{\odot}$ (the core of a $25 M_{\odot}$ star) is exploded with a kinetic energy $E_{\mathrm{K}}=1.0 \times 10^{51} \mathrm{erg}$ and an ejecta mass $M_{\mathrm{ej}}=4.6 M_{\odot}$. For the hypernova model $(\mathrm{CO} 100)$, a $\mathrm{C}+\mathrm{O}$ star of $M_{\mathrm{CO}}=10.0 M_{\odot}$ (the core of a $30-35 M_{\odot}$ star) is exploded with $E_{\mathrm{K}}=8.0 \times 10^{51} \mathrm{erg}$ and $M_{\mathrm{ej}}=8.0 M_{\odot}$. The mass of ${ }^{56} \mathrm{Ni}$ is set to be $0.15 M_{\odot}$ for both models to explain the observed peak brightness.

In Figure 1, we compare the synthetic $V$ light curves for models $\mathrm{CO} 60$ and CO100 with the $V$ light curve of SN 1997ef. A distance modulus of $\mu=33.6 \mathrm{mag}$ and a color excess $E(B-V)=0.00$ are adopted. Both models give synthetic light curves with quite a broad peak and a relatively slow tail, which reproduce the light curve of SN $1997 \mathrm{ef}$ reasonably well. We should note that the model light curve declines somewhat faster than the observed tails of SN 1997ef and, 


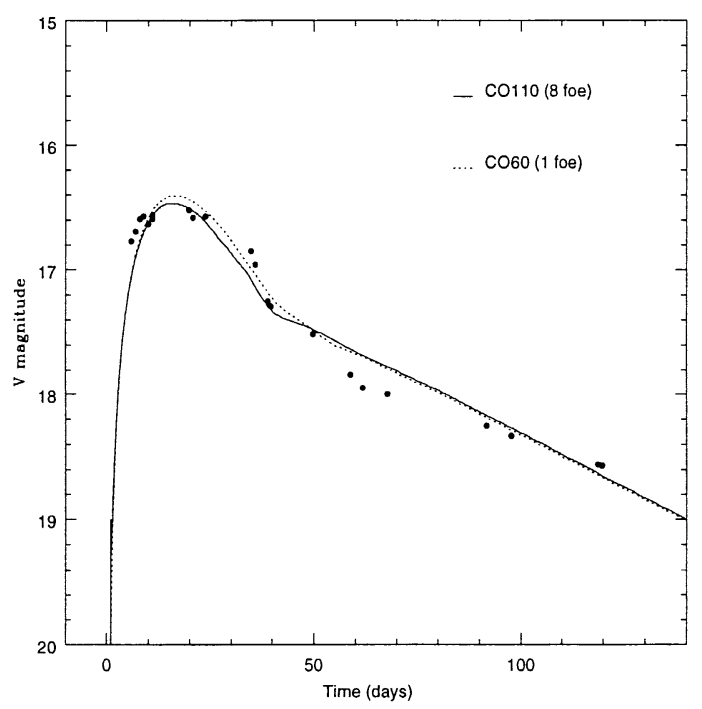

Figure 1. The $V$ light curves of models $\mathrm{CO} 100$ (solid line) and $\mathrm{CO} 60$ (dashed line) compared with the observations of SN 1997ef. A distance modulus of $\mu=33.6 \mathrm{mag}$ is adopted.

as shown later, of SN $1998 \mathrm{bw}$. We will see in the next section that this might be due to some asymmetry in the explosion.

Light curve modeling provides important constraints on $M_{\mathrm{CO}}$ and $E_{\mathrm{K}}$. However, it is difficult to distinguish clearly between the ordinary SN Ic and the hypernova model from the light curve alone, since models with different values of $M_{\mathrm{ej}}$ and $E_{\mathrm{K}}$ can reproduce the observed light curve equally well if $M_{\mathrm{ej}}^{3 / 4} E_{\mathrm{K}}^{-1 / 4}=$ const. On the other hand, these models can be expected to produce different spectra because of the different $E_{\mathrm{K}}$. Therefore, spectrum synthesis can tell these models apart, taking advantage of their differences.

Based on the hydrodynamical models $\mathrm{CO} 60$ and CO100, i.e., using their density structure and composition, we computed synthetic spectra using a Monte Carlo spectrum-synthesis code (Mazzali \& Lucy 1993; Lucy 2000; Mazzali 2000). Spectra computed with model CO60 for the first three epochs are compared to the observed spectra in Figure 2 (left). All synthetic spectra show narrow lines, much narrower than observed. This clearly indicates a lack of material at high velocity in model CO60 and suggests that the kinetic energy of this model is too small. Synthetic spectra computed with model CO100 for the same three epochs are also shown in Figure 2 (right). The spectra have much broader lines and are in good agreement with the observations.

Since the spectra of SN 1997ef are reproduced much better with the energetic model CO100 than with the ordinary SN Ic model CO60, we conclude that SN 1997ef is a hypernova. 

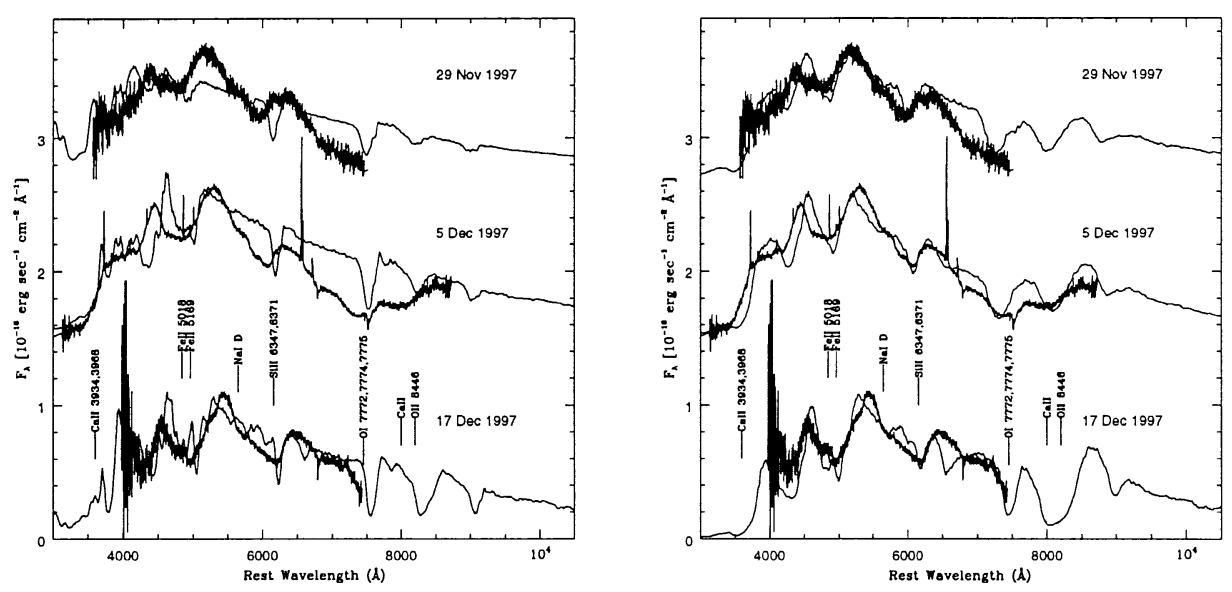

Figure 2. Left: Observed spectra of SN 1997ef (bold lines) and synthetic spectra computed using model $\mathrm{CO} 60$. The lines in the synthetic spectra are much too narrow. Right: Observed spectra of SN 1997ef (bold lines) and synthetic spectra computed using model CO100 (fully drawn lines).

\section{SN $1998 \mathrm{bw}$}

SN 1998bw is very likely connected with GRB 980425 (Galama et al. 1998; Kulkarni et al. 1998). This object was soon identified to be a supernova from its light curve, which was different from those of typical optical transients of gamma-ray bursts (Galama et al. 1998; Patat et al. 2000b). Early spectra were rather blue and featureless, quite unlike any other known SNe. However, more careful inspection showed striking similarities with the spectra of Type Ic SNe, but with one major difference: the absorption lines are so broad in SN 1998bw that they blend together, giving rise to what could even be confused with an emission spectrum. Velocities in the Si II $6355 \AA$ line are as high as $30,000 \mathrm{~km} \mathrm{~s}^{-1}$. Also, the SN was very bright for a SN Ic: the observed peak luminosity, $L \sim 1.4 \times 10^{43} \mathrm{erg} \mathrm{s}^{-1}$, is almost ten times higher than that of previously known $\mathrm{SNe} \mathrm{Ib/Ic} \mathrm{(Iwamoto} \mathrm{et}$ al. 1998; Woosley, Eastman, \& Schmidt 1999).

We calculate the light curves and spectra for various $\mathrm{C}+\mathrm{O}$ star models with different values of $E_{\mathrm{K}}$ and $M_{\mathrm{ej}}$. These parameters can be constrained by comparing the calculated light curves, the synthetic spectra, and the photospheric velocities with the data of SN 1998bw. Here, we describe the best model, which is the explosion of a $13.8 M_{\odot} \mathrm{C}+\mathrm{O}$ star with a large kinetic energy $\left(E_{\mathrm{K}}=6 \times 10^{52} \mathrm{erg}\right)$. Such a massive $\mathrm{C}+\mathrm{O}$ star progenitor is the product of a main-sequence star of $\sim 40 M_{\odot}$ which lost its $\mathrm{H} / \mathrm{He}$ envelope via a stellar wind or binary interaction. For a distance modulus of $\mu=32.89 \mathrm{mag}$, a ${ }^{56} \mathrm{Ni}$ mass of $\sim 0.63 M_{\odot}$ is necessary to reproduce the light-curve maximum. Figure 3 shows the observed spectra of SN 1998bw (solid lines) and synthetic spectra computed using model CO138 (dashed lines). We can see that the broad lines are well reproduced because we have enough material at high velocities in this model. 
Despite the success of our "hypernova" model in reproducing the early light curve of SN $1998 \mathrm{bw}(t \lesssim 60$ days), the model light-curve tail declines more rapidly than the observations do (Figure 4). After $t \sim 200$ days, the decline of the model light curve becomes slower, and it approaches the half-life of ${ }^{56}$ Co decay around Day 400. At $t \gtrsim 400$ days, most $\gamma$-rays escape from the ejecta, while positrons emitted from the ${ }^{56} \mathrm{Co}$ decay are mostly trapped and their energies are thermalized. Therefore, positron deposition determines the light curve at $t \gtrsim 400$ days. If the observed tail should follow the positron-powered light curve, the ${ }^{56}$ Co mass could be determined directly.

The deviation of the model light curve from the observation at $t \gtrsim 60$ days suggests that there might be a high-density region in the ejecta of SN $1998 \mathrm{bw}$ where the $\gamma$-rays are efficiently trapped. Another unexpected feature appeared in the late-phase spectra. Measuring the velocity of each element (Patat et al. 2000a), we note that iron expands faster than oxygen, which is contrary to expectations.

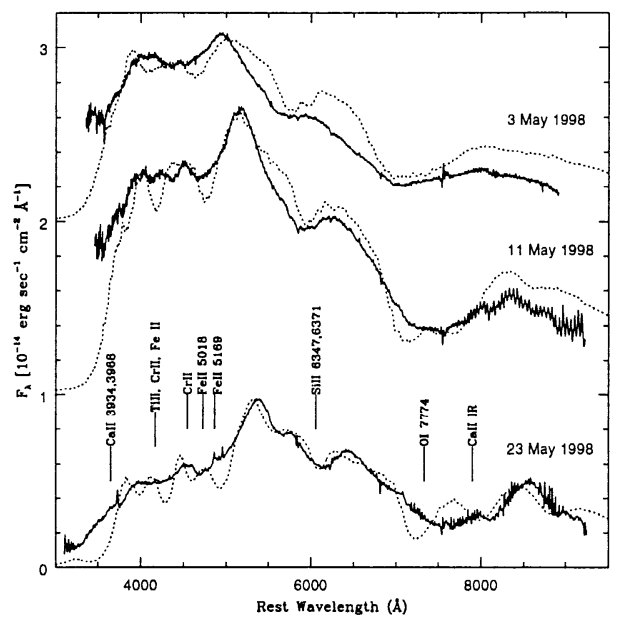

Figure 3. Observed spectra of SN 1998bw (full lines) and synthetic spectra computed using model CO138 (dashed lines).

Both the need for a high-density region and the velocity inversion might indicate that the explosion is aspherical (Höflich, Wheeler, \& Wang 1999; MacFadyen \& Woosley 1999). If the outburst in SN 1998bw took the form of a prolate spheroid, as also indicated by polarization measurements (Patat et al. $2000 \mathrm{~b}$ ), the explosive shock along the long axis was probably strong, ejecting material with large velocities and producing abundant ${ }^{56} \mathrm{Ni}$. In directions away from the long axis, on the other hand, oxygen is not consumed and the density is high enough for $\gamma$-rays to be trapped even at advanced phases, thus giving rise to the slowly declining tail (see $§ 5$ ). 


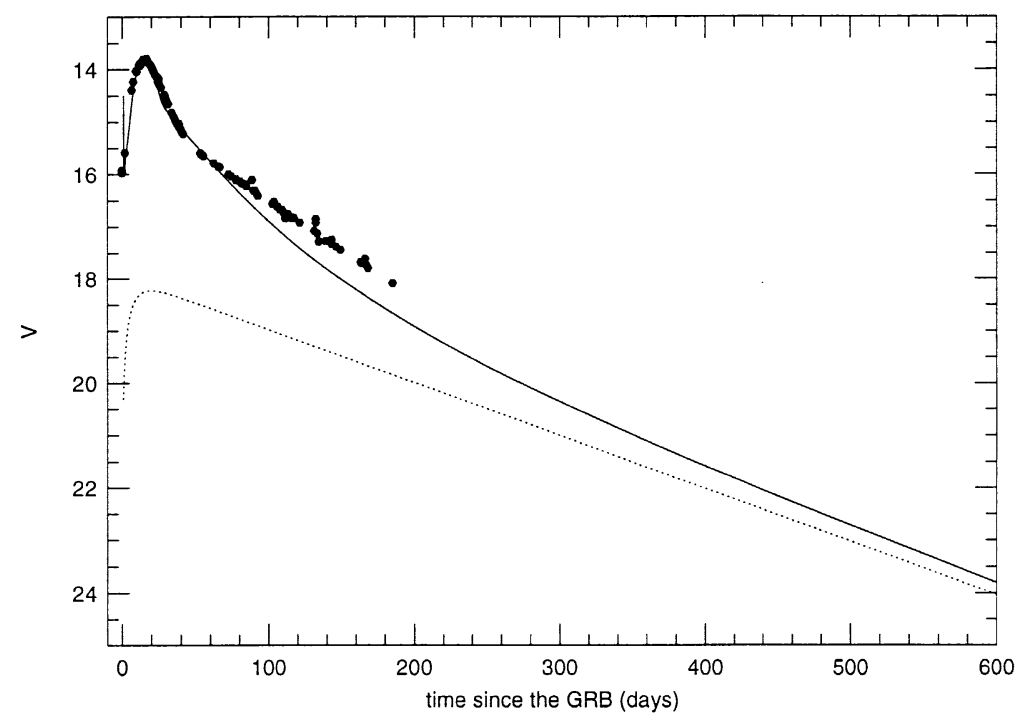

Figure 4. The light curve of model CO138 compared with the observations of SN 1998bw. A distance modulus of $\mu=32.89 \mathrm{mag}$ is adopted. The dotted line indicates the energy deposited by positrons.

\section{SN1997cy}

SN 1997cy was discovered on July 16, 1997 in the compact and faint galaxy of the Abell 3266 Cluster at a distance modulus of $\mu=37.40$ (Germany et al. 2000). The SN displays narrow $\mathrm{H} \alpha$ emission, which led to the classification of SN 1997cy as a Type IIn. It is noteworthy that the epoch of the SN explosion, constrained by a prediscovery limit on March 12 , is consistent with the association of the SN with GRB 970514 (Germany et al. 2000).

SN 1997cy is the brightest SN II discovered so far (Turatto et al. 2000; Figure 5 ). Its light curve does not conform to the classical templates of SNe II (namely, Plateau and Linear) but resembles the slow evolution of the Type IIn SN 1988 Z (Turatto et al. 1993). SN 1997cy could be powered by either the radioactive decay of ${ }^{56} \mathrm{Ni}$ to ${ }^{56} \mathrm{Co}$ and ${ }^{56} \mathrm{Fe}$, or by circumstellar interaction. After about Day 120 , the SN light decline becomes slower than the lifetime of ${ }^{56} \mathrm{Co}$, suggesting circumstellar interaction for the energy source.

In the interaction model, collision of the SN ejecta with the slowly moving circumstellar matter (CSM) converts the kinetic energy of the ejecta into light, thus producing the observed, intense light display of the SN. Our exploratory model considers the explosion of a massive star of $M=25 M_{\odot}$ with a parameterized kinetic energy $E_{\mathrm{K}}$. We assume that the collision starts near the stellar radius at a distance $r_{1}$ where the density of the CSM is $\rho_{1}$, and we adopt for the 


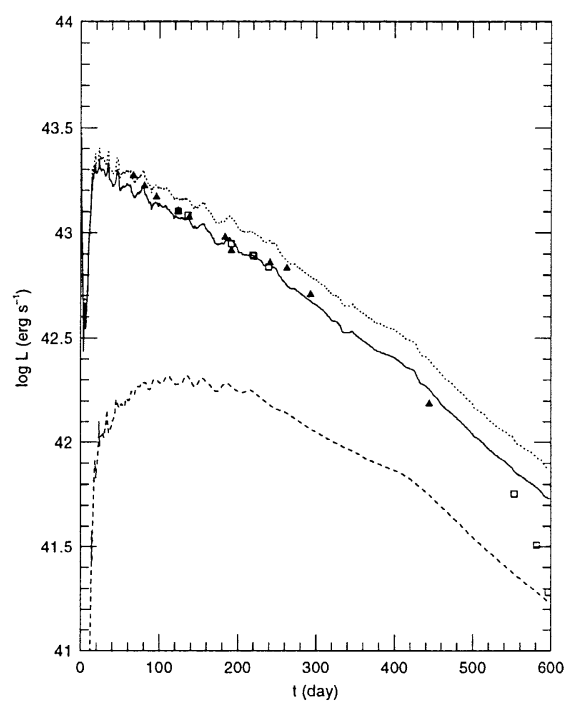

Figure 5. The uvoir bolometric light curve of SN 1997cy compared with the synthetic light curve obtained with the CSM interaction model.

CSM a power-law for the density profile $\rho \propto r^{n}$. The parameters $E_{\mathrm{K}}, \rho_{1}$, and $n$ are constrained by comparison with the observations.

The regions excited by the forward and reverse shocks emit mostly X-rays. The density in the shocked ejecta is so high that the reverse shock is radiative and a dense cooling shell is formed (e.g., see Suzuki \& Nomoto 1995; Terlevich et al. 1992). The X-rays are absorbed by the outer layers and core of the ejecta, and re-emitted as UV-optical photons.

Figure 5 shows the model light curve which best fits the observations. The model parameters are: $E_{\mathrm{K}}=5 \times 10^{52} \mathrm{erg}, \rho_{1}=4 \times 10^{-14} \mathrm{~g} \mathrm{~cm}^{-3}$ at $r_{1}=$ $2 \times 10^{14} \mathrm{~cm}$ (which corresponds to a mass-loss rate of $\dot{M}=4 \times 10^{-4} M_{\odot} \mathrm{yr}^{-1}$ for a wind velocity of $10 \mathrm{~km} \mathrm{~s}^{-1}$ ), and $n=-1.6$. The large mass-loss episode giving rise to the dense CSM is supposed to have occurred after the progenitor made a loop in the HR diagram from BSG to RSG. In this model, the mass of the low-velocity CSM is $\sim 5 M_{\odot}$, which implies that the transition from BSG to RSG took place about $10^{4} \mathrm{yr}$ before the SN event.

The large CSM mass and density, as well as the very large explosion energy, are necessary to have large, shocked masses and thus to reproduce the observed high luminosity. For models with low $E_{\mathrm{K}}$ and high $\rho_{1}$, the reverse-shock speed is too low to produce a sufficiently high luminosity. For example, a model with $E_{\mathrm{K}}=10^{52} \mathrm{erg}$ and $\rho_{1}$ as above yields a value of $L_{\mathrm{UVOIR}}$ lower than the observed luminosity by a factor of $\sim 5$. For high $E_{\mathrm{K}}$ or low $\rho_{1}$, the expansion of the SN ejecta is too fast for the cooling shell to absorb enough X-rays to sustain the luminosity. Thus, in this model, $E_{\mathrm{K}}$ and $\dot{M}$ are constrained within a factor of $\sim 3$ of the reported values.

The shape of the light curve constrains the circumstellar density structure. For $n=-2$, the case of a steady wind, $L_{\mathrm{UVO}}$ decreases too rapidly around Day 
200. To reproduce the observed decrease after $t \sim 300$ days, the CSM density is assumed to drop abruptly at the radius the forward shock reaches at Day 300 , so that the collision becomes weaker afterwards. (Such a change of the CSM density corresponds to the transition from BSG to RSG of the progenitor $\sim 10^{4} \mathrm{yr}$ before the SN explosion.) After Day 550, the observed light curve drops more sharply than the model, which might indicate the development of clumps in the ejecta and a resulting decrease in the effective X-ray absorption.

The ejecta-CSM model with high explosion energy is thus able to reproduce the light curve. However, this does not necessarily mean that the radioactive energy input is small, because of the uncertainty in the model fitting. To constrain the contribution from the radioactive decay, we calculate the optical light curve of the SN including shock heating and radioactive decay with various amount of ${ }^{56} \mathrm{Ni}$. We find that a contribution of at most $0.7 M_{\odot}$ of ${ }^{56} \mathrm{Ni}$ is allowed. We also note that the decline rate of the radioactive component is much faster than the half-life of the ${ }^{56} \mathrm{Co}$ decay because the explosion is so energetic that only a fraction of the $\gamma$-rays is trapped in the ejecta. This implies that radioactive decay cannot explain the slope of the observed light curve even between 60 and 120 days.

Its large kinetic energy gains SN 1997cy enrollment in the family of "hypernovae". While SN 1998bw and SN 1997ef are Type Ic, SN 1997cy is Type IIn. The recent SN 1999E (Turatto et al. 2000), whose spectrum is identical to that of SN 1997cy, may be another Type IIn hypernova and so may be SN 1988Z if a new study should lead to larger estimates for the mass and kinetic energy.

\section{Nucleosynthesis in Asymmetric Explosions}

In $\S 3$, we speculate that the expansion velocity of $\mathrm{Fe}$ and $\mathrm{O}$ in SN 1998bw betrays the effect of the asymmetry in the explosion. To confirm this, we calculate the explosive nucleosynthesis in an axisymmetric explosion. Our calculations are performed in two steps. The first step is a hydrodynamical simulation of the explosions with a two-dimensional, hydrodynamical code (Hachisu et al. 1991; Nagataki et al. 1997). In this calculation, we follow tracks of a number of test particles and their density and temperature history. This history is used in the second step to calculate the change in the chemical composition with a reaction network including a total of 222 isotopes up to ${ }^{71} \mathrm{Ge}$ (Thielemann, Nomoto, \& Hashimoto 1996).

Figure 6 shows the isotopic compositions of the ejecta of the axisymmetric explosion in the direction of the jet (top) and perpendicular to the jet (bottom). The progenitor model is the $16 M_{\odot}$ He core of a $40 M_{\odot}$ star (Nomoto et al. 1997; Nomoto \& Hashimoto 1988). The explosion energy is $E_{\mathrm{K}}=1 \times 10^{51} \mathrm{erg}$. Starting the hydrodynamical simulation, we deposit the energy as $50 \%$ thermal energy and $50 \%$ kinetic energy toward the jet $(z)$ below the mass cut that divides the ejecta and the collapsing core.

The shock is stronger, and the post-shock temperatures are higher, along the jet direction $(z)$, so that explosive nucleosynthesis takes place in a more extended, lower-density region compared with the perpendicular direction $(r)$. A larger amount of ${ }^{56} \mathrm{Ni}$, which decays into ${ }^{56} \mathrm{Fe}$ through ${ }^{56} \mathrm{Co}$, is produced in the jet direction. In addition, elements produced by $\alpha$-rich freezeout are enhanced 

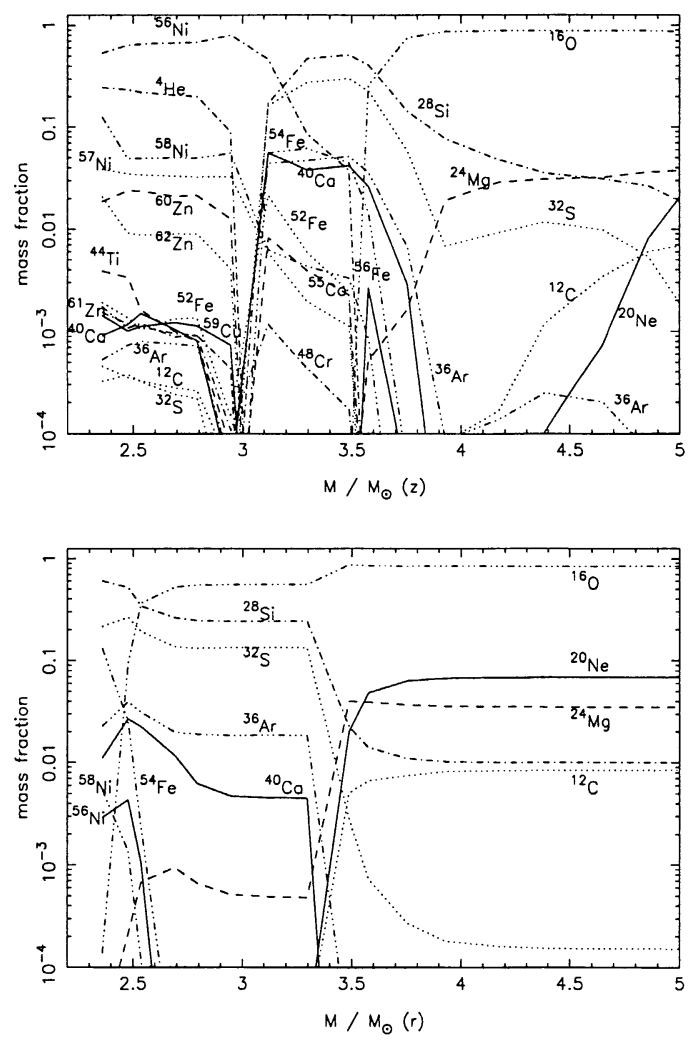

Figure 6. The isotopic compositions of the ejecta of the axisymmetric explosion in the direction of the jet (top) and perpendicular to the jet (bottom).

because nucleosynthesis proceeds at higher entropies than in the region outside the jet. Figure 6 clearly shows that in the jet direction a larger amount of ${ }^{4} \mathrm{He}$ is left after the shock decomposition. Hence, elements synthesized through capturing $\alpha$-particles, such as ${ }^{44} \mathrm{Ti}$ and ${ }^{48} \mathrm{Cr}$ (decaying into ${ }^{44} \mathrm{Ca}$ and ${ }^{48} \mathrm{Ti}$, respectively), become more abundant. Our results are consistent with previous, similar calculations (Nagataki et al. 1997). In contrast, little ${ }^{56} \mathrm{Ni}$ is produced in the $r$-direction. Also, the expansion velocities are lower than those in the $z$-direction. Therefore, the Fe velocities (mostly in the $z$-direction) can exceed the $\mathrm{O}$ velocities (in the $r$-direction), as observed in SN 1998bw. Oxygen in the $z$-direction has the highest velocities, but the densities may become too low to be excited by gamma-rays.

The above nucleosynthesis model might shed light on a problem in the Galactic chemical-evolution model. It has been pointed out that $\mathrm{Ti}$ is deficient in Galactic chemical-evolution models with the usual supernova yields (Timmes, Woosley, \& Weaver 1995; Ikuta 1998). However, if the contribution from hy- 
pernovae to the Galactic chemical evolution is relatively large, this problem could be relaxed. As we have seen, $\alpha$-rich freezeout is enhanced in axisymmetric explosions, and $\mathrm{Ti}$ is produced more abundantly. This possibility should be investigated further, together with more extensive nucleosynthesis calculations.

\section{Summary}

We have investigated the three peculiar supernovae SN Ic 1997ef, SN Ic 1998bw, and SN IIn 1997cy. We have shown that all of them have much larger explosion energies $\left(E_{\mathrm{K}} \gtrsim 10^{52} \mathrm{erg}\right)$ than usual supernovae $\left(E_{\mathrm{K}} \sim 10^{51} \mathrm{erg}\right)$, which leads us to call them "hypernovae". We have also suggested some degree of aspherical explosions for SN 1997ef and SN 1998bw from the comparison between our models and the observations. The polarization of SN 1998bw $(\lesssim 1 \%)$ indicates an axial ratio of 2:1 (Höflich et al. 1999). Thus, our spherical models may overestimate $E_{\mathrm{K}}$ by a factor of a few, but the $E_{\mathrm{K}} \mathrm{s}$ still are unusually large.

One may note that they all are candidates for GRB/SNe connection. SN Ic 1997 ef might have been connected with GRB 971115, SN Ic 1998bw with GRB 980425, and SN IIn 1997cy with GRB 970514. There are two other high-redshift GRBs which might have been associated with a SN: GRB 980326 (Bloom et al. 1999) and GRB 970228 (Reichart 1999; Galama et al. 2000). To confirm these GRB/SN connections, relativistic, asymmetric explosion models should be constructed (e.g., Aloy et al. 1999; MacFadyen \& Woosley 1999).

\section{References}

Aloy, M. A., Ibáñez, J. M. A., Martí, J. M. A., Gómez, J.-L., \& Müller, E. 1999, ApJ, 523, L125

Bloom, J. S. et al. 1999, Nature, 401, 453

Filippenko, A. V., et al. 1997, IAU Circ. No. 6783, 6809

Galama, T. J., et al. 1998, Nature, 395, 670

Galama, T. J., et al. 2000, ApJ, submitted

Garnavich, P., et al. 1997, IAU Circ. No. 6778, 6786, 6798

Germany, L. M., Reiss, D. J., Sadler, E. M., Schmidt, B. P., \& Stubbs, C. W. 2000, ApJ, submitted

Hachisu, I., Matsuda, T., Nomoto, K., \& Shigeyama, T. 1991, ApJ, 368, L27

Höflich, P., Wheeler, J. C., \& Wang, L. 1999, ApJ, 521, 179

Ikuta, C. 1998, M.A. thesis, University of Tokyo

Iwamoto, K., et al. 1998, Nature, 395, 672

Iwamoto, K., et al. 2000, ApJ, in press

Kulkarni, S. R., et al. 1998, Nature, 395, 663

Lucy, L. B. 2000, A\&A, in press

MacFadyen, A. I., \& Woosley, S. E. 1999, ApJ, 524, 262

Mazzali, P. A. 2000, A\&A, submitted

Mazzali, P. A., \& Lucy, L. B. 1993, A\&A, 279, 447 
Nagataki, S., Hashimoto, M.-A., Sato, K., \& Yamada, S. 1997, ApJ, 486, 1026

Nomoto, K., \& Hashimoto, M. 1988, Phys. Rep., 256, 173

Nomoto, K., Yamaoka, H., Pols, O. R., van den Heuvel, E. P. J., Iwamoto, K., Kumagai, S., \& Shigeyama, T. 1994, Nature, 371, 227

Nomoto, K., et al. 1997, Nucl. Phys. A, 616, 79

Patat, F., et al. 2000a, Mem. Soc. Astron. Italy, in press

Patat, F., et al. 2000b, ApJ, submitted

Reichart, D. E. 1999, ApJ, 521, L111

Shigeyama, T., \& Nomoto, K. 1990, ApJ, 360, 242

Suzuki, T., \& Nomoto, K. 1995, ApJ, 455, 658

Terlevich, R., Tenorio-Tagle, G., Franco, J., \& Melnick, J. 1992, MNRAS, 255, 713

Thielemann, F.-K., Nomoto, K., \& Hashimoto, M. 1996, ApJ, 460, 408

Timmes, F. X., Woosley, S. E., \& Weaver, T. A. 1995, ApJS, 98, 617

Turatto, M., Cappellaro, E., Danziger, I. J., Benetti, S., Gouiffes, C., \& della Valle, M. 1993, MNRAS, 262, 128

Turatto, M., et al. 2000, ApJ, submitted

Wang, L.-F., \& Wheeler, J. C. 1998, ApJ, 504, L87

Woosley, S. E., Eastman, R. G., \& Schmidt, B. P. 1999, ApJ, 516, 788 